\title{
Vortex pump for dilute Bose-Einstein condensates
}

\author{
Mikko Möttönen, ${ }^{1,2}$ Ville Pietilä, ${ }^{1}$ and Sami M. M. Virtanen ${ }^{1}$ \\ ${ }^{1}$ Laboratory of Physics, Helsinki University of Technology P. O. Box 4100, FI-02015 TKK, Finland \\ ${ }^{2}$ Low Temperature Laboratory, Helsinki University of Technology, P.O. Box 3500, 02015 TKK, Finland
}

\begin{abstract}
The formation of vortices by topological phase engineering has been realized experimentally to create the first two- and four-quantum vortices in dilute atomic Bose-Einstein condensates [A. E. Leanhardt et al., Phys. Rev. Lett. 89, 190403 (2002)]. We consider a similar system, but in addition to the Ioffe-Pritchard magnetic trap we employ an additional hexapole field. By controlling cyclically the strengths of these magnetic fields, we show that a fixed amount of vorticity can be added to the condensate in each cycle. In an adiabatic operation of this vortex pump, the appearance of vortices into the condensate is interpreted as the accumulation of a local Berry phase. Our design can be used as an experimentally realizable vortex source for possible vortex-based applications of dilute Bose-Einstein condensates.
\end{abstract}

PACS numbers:

Quantized vortices manifest the long-range phase coherence of many-particle quantum systems which are described by a complex-valued order parameter field, and their existence and stability is intimately related to the superfluid properties of the system [1]. In a loop encircling the vortex, the phase of the order parameter undergoes an integer multiple $\kappa$ of $2 \pi$ winding. This number $\kappa$ is the circulation quantum number of the vortex.

The first vortices in trapped gaseous Bose-Einstein condensates (BECs) of atoms were created by driving dynamically a transition between two different hyperfine spin states of the condensed atoms with a rotating laser field [2], as suggested in Ref. [3]. Single-quantum vortices have also been nucleated by slicing through the condensate with a focused laser beam moving faster than the critical velocity [4], by colliding condensates separated by tailored optical potentials [5], by stirring the condensate with a laser beam [6], and by rotating the condensate with an asymmetric trapping potential [7]. In harmonic traps, multi-quantum vortices are unstable against splitting into single-quantum vortices [8, 9, 10], and hence rotation of the condensate with frequencies close to the harmonic trapping frequency results in triangular vortex lattices of single-quantum vortices [11, 12]. Recently, multi-quantum vortices have been created by a coherent transfer of angular momentum from a photon of a Laguerre-Gaussian laser beam to the condensate 13]. Furthermore, giant vortices with circulations up to 60 quanta have been created from such vortex lattices with a tightly focused laser removing atoms from the center of the condensate for the giant vortex to appear [14].

In contrast to the above-mentioned dynamical methods, vortices can also be created with the so-called topological phase engineering technique [15, 16, 17, 18]. Here, the phase winding of the vortex is generated by an azimuthally dependent adiabatic turn of the hyperfine spin of the condensate. The first two- and four-quantum vortices were created in dilute BECs with this method by Leanhardt et al. [19]. They reversed the bias field of the Ioffe-Pritchard trap and verified that the resulting twoand four-quantum vortex states were consistent with the angular momenta of $2 \hbar$ and $4 \hbar$ per particle, respectively. After the reversal, the bias magnetic field was brought back to its initial configuration and the topological unwinding of the vortices was observed.

In this Letter, we show that by controlling the strengths of the bias and the quadrupole fields of the Ioffe-Pritchard trap and an additional hexapole magnetic field, vortex pumping can be implemented, i.e., the quantum number of the vortex in the condensate is increased by a constant amount in each pumping cycle. The pumping can be carried out either fully adiabatically or partly adiabatically and partly instantaneously. The operation of the pump is studied analytically within the adiabatic approximation, and computationally using the Gross-Pitaevskii (GP) equation for hyperfine spin $F=1$ condensates. In this special case, the reversal of the bias field in the presence of the hexapole field creates a fourquantum vortex. When the bias field is brought back to its initial configuration in the presence of the quadrupole field, two quanta of vorticity is lost. Thus the pump creates two net quanta of vorticity per cycle. To date, the vortex pump introduced here is the only known method to produce almost any desired amount of vorticity without particle loss. Furthermore, the achieved vortex densities can possibly be high enough for the experimental realisation of strongly correlated vortex liquid phases [20].

In the adiabatic approximation for an atom with a positive Landé $g$ factor, the spin of the condensate is antiparallel to the local magnetic field $\boldsymbol{B}=\boldsymbol{B}_{\perp}+\boldsymbol{B}_{z}$, where $\boldsymbol{B}_{z}$ is the uniform bias field along the positive $z$-direction and the perpendicular part $\boldsymbol{B}_{\perp}$ is either the quadrupole or the hexapole field given in the polar coordinates by

$$
\begin{aligned}
\boldsymbol{B}_{q}(r, \phi, t) & =B_{q}^{\prime}(t) r[\cos (\phi) \hat{\boldsymbol{x}}-\sin (\phi) \hat{\boldsymbol{y}}], \\
\boldsymbol{B}_{h}(r, \phi, t) & =B_{h}^{\prime}(t) r[\cos (2 \phi) \hat{\boldsymbol{x}}-\sin (2 \phi) \hat{\boldsymbol{y}}],
\end{aligned}
$$

respectively. Here, $\hat{\boldsymbol{x}}$ and $\hat{\boldsymbol{y}}$ are the unit vectors along the positive $x$ - and $y$-directions, respectively. The above fields up to quadratic terms in the coordinates can be realized experimentally with five coils in total and the field strengths $B_{q}^{\prime}(t)$ and $B_{h}^{\prime}(t)$ can be controlled by changing the currents in the coils [1]. 
Let us assume that the adiabatic approximation is valid and the bias field $\boldsymbol{B}_{z}$ is initially dominating over the perpendicular field such that the spin of the condensate is aligned with the $z$-axis, i.e., is in the weak-field-seeking state $\left|F, m_{z}=-F\right\rangle$, where $m_{z}$ is the quantum number of the spin along the $z$-direction. Thus the reversal of the bias field introduces a spin rotation about the normal vector $\hat{\boldsymbol{n}}$ of the perpendicular field in the $x y$-plane as

$$
R_{\hat{\boldsymbol{n}}}(\pi)=e^{-i \pi \mathcal{F} \cdot \hat{\boldsymbol{n}}},
$$

where the dot product is defined as $\mathcal{F} \cdot \hat{\boldsymbol{n}}=\mathcal{F}_{x} n_{x}+$ $\mathcal{F}_{y} n_{y}+\mathcal{F}_{z} n_{z}$ and $\mathcal{F}_{\alpha}$ is the dimensionless spin operator in the direction $\alpha$. From Eqs. (11) and (2) we obtain the normal vectors

$$
\begin{aligned}
\hat{\boldsymbol{n}}_{q}(\phi) & =\sin (\phi) \hat{\boldsymbol{x}}+\cos (\phi) \hat{\boldsymbol{y}}, \\
\hat{\boldsymbol{n}}_{h}(\phi) & =\sin (2 \phi) \hat{\boldsymbol{x}}+\cos (2 \phi) \hat{\boldsymbol{y}},
\end{aligned}
$$

for the quadrupole and hexapole fields, respectively. Thus the reversal of the bias field in the presence of the hexapole field steers the initial spin state to

$$
R_{\hat{\boldsymbol{n}}_{h}}(\pi)\left|F, m_{z}\right\rangle=(-1)^{F+m_{z}} e^{-i 4 m_{z} \phi}\left|F,-m_{z}\right\rangle,
$$

changing the vorticity of the system by $-4 m_{z}$. The above result can be obtained by expressing the rotation with the Euler angles as $R_{\hat{\boldsymbol{n}}_{h}}(\pi)=R_{\hat{\boldsymbol{z}}}(\pi / 2-2 \phi) R_{\hat{\boldsymbol{x}}}(\pi) R_{\hat{\boldsymbol{z}}}(2 \phi-$ $\pi / 2)$.

These results on the reversal of the bias field are the basis of topological phase engineering to create vortices [15, 16, 17, 18]. In vortex pumping, however, the control sequence is to be cyclic, and hence we have to turn the spin back to its original position to end the cycle. If this is carried out with the same perpendicular field as the first reversal, Eq. (3) implies that the two reversals cancel each other, $R_{\hat{\boldsymbol{n}}}(-\pi) R_{\hat{\boldsymbol{n}}}(\pi)=I$, and the topologically created vortex unwinds as was observed in Ref. [19]. In general, only one control parameter cannot provide geometric effects in cyclic and adiabatic temporal evolution. Furthermore, since the spin rotation axis in the bias field reversal does not depend on the absolute strength of the perpendicular field and the reversal of the perpendicular field before a subsequent reversal of the bias field results in $R_{-\hat{\boldsymbol{n}}}(-\pi) R_{\hat{\boldsymbol{n}}}(\pi)=R_{\hat{\boldsymbol{n}}}(2 \pi)=I$ for bosons, two different perpendicular fields are required for a feasible pumping cycle. Such a cycle is shown in Fig. 1 for our pumping scheme. In fact, the paths between the initial and the middle point of the cycle can be arbitrary with the constraint that either $B_{q}^{\prime}$ or $B_{h}^{\prime}$ vanishes always in the adiabatic evolution.

We begin from the ground state of the system which corresponds to

$$
\left\langle\boldsymbol{r}, F, m_{z} \mid \Psi_{0}\right\rangle=\sqrt{\rho(\boldsymbol{r})} \delta_{m_{z},-F},
$$

where $\rho(\boldsymbol{r})$ is the condensate density and $\delta_{i, j}$ the Kronecker delta [26]. By adiabatically ramping up the hexapole field, reversing the bias field, and ramping down the hexapole field, we have performed the spin rotation corresponding to Eq. (6), and achieved the $4 F$-quantum vortex state $e^{i 4 F \phi} \sqrt{\rho^{(1)}(\boldsymbol{r})}\left|F, m_{z}=F\right\rangle$, where the superscript denotes possible changes in the particle density due to the emergence of the vortex. Then, we bring the spin state back to its initial position by adiabatically ramping up the quadrupole field, reversing again the bias field, and ramping down the quadrupole field. This corresponds to the spin rotation $R_{\hat{\boldsymbol{n}}_{q}}(-\pi)$ which removes $2 F$ quanta of vorticity. Thus the total effect of $n$ adiabatic pumping cycles on the initial ground state is

$$
\left\langle\boldsymbol{r}, F, m_{z} \mid \Psi_{n}\right\rangle=e^{i 2 n F \phi} \sqrt{\rho^{(2 n)}(\boldsymbol{r})} \delta_{m_{z},-F} .
$$

We observe that our pump increases the vorticity of the system by $2 F$ per cycle. Since the particle density in this trapping scenario is independent of the azimuthal angle $\phi$, the axial angular momentum corresponding to this vorticity is $\left\langle\Psi_{n}\left|\hat{L}_{z}\right| \Psi_{n}\right\rangle=2 n N F \hbar$, where $N=\int \rho(\boldsymbol{r}) \mathrm{d} \boldsymbol{r}$ is the total particle number.
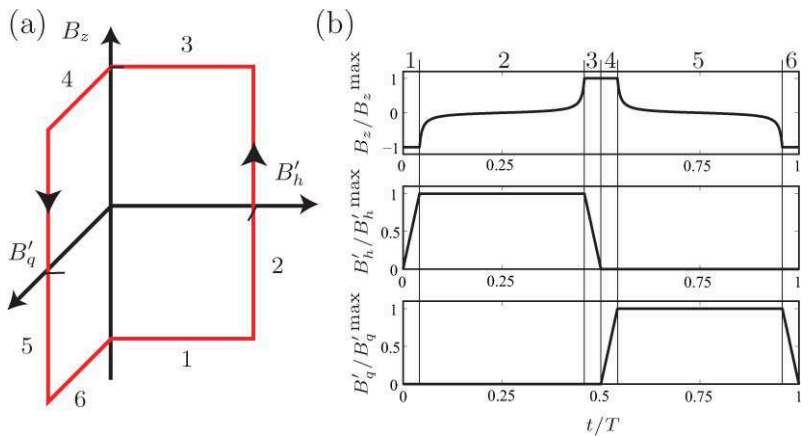

FIG. 1: (color online). Possible control cycle in the magnetic field parameter space for pumping vortices into a spinor BoseEinstein condensate (a). Panel (b) displays an example of the temporal changes of the control fields such that the spin at a certain distance from the $z$-axis is reversed with a constant speed. The different parts of the control loop in panel (a) are marked in the top of panel (b).

The topological phase engineering method has been explained in terms of accumulation of geometric phases [21] for individual spins of the atoms the condensate is composed of 17, 19]. This interpretation is based on the result by Berry [21] that an adiabatically and cyclically turned spin acquires a geometric phase equal to $-m_{F} \Omega$, where $\Omega$ is the solid angle covered by the path of the spin with the initial state $\left|F, m_{F}\right\rangle$. The result of Eq. (6) follows from the fact that the solid angle covered by the paths of two different spins separated by an angle $\theta$ in the $\phi$ coordinate is $4 \theta$ for the reversal of the bias field in the presence of the hexapole field. In the case of the vortex pump, the result follows directly from Berry's argument, since we need only to consider the path traced by a single spin. In our pumping scheme, the solid angle $\Omega$ equals twice the angle between the normal vectors of the hexapole and the quadrupole fields given in 
Eqs. (4) and (5). This results in the local Berry phase of $2 \phi$, consistent with pumping two quanta of vorticity per cycle. Note that the above interpretation of vortex pumping in terms of the Berry phase is not directly related to the usual Berry phase accumulated to a macroscopic quantum state of a system during adiabatic control parameter cycles.

The adiabatic turning of the condensate spin by the magnetic field requires that the magnetic energy dominates over the kinetic energy at each spatial point and that the field is rotated slowly enough to avoid spin flips due to Landau-Zener transitions. Thus to guarantee adiabaticity, the strength of the external magnetic field $|\boldsymbol{B}(\boldsymbol{r})|$ should be large enough in the condensate region. However, this condition is not fulfilled on the $z$-axis when the bias field crosses zero, and hence it is desirable to avoid particles from entering this area. This can be achieved by introducing an additional optical plug potential

$$
V_{\text {plug }}(r)=A e^{-r^{2} / \delta^{2}},
$$

where $A$ is the strength of the plug and $\delta$ its width. The plug can be realized for pancake-shaped condensates by a focused laser beam as was done, e.g., in Ref. 222]. In addition, to improve adiabaticity during the magnetic field field reversal, the optical plug tends to stabilize the created multi-quantum vortex against splitting.

The magnetic trapping potential arises due to the spatial dependence of the perpendicular field. Thus the magnetic trap vanishes in the beginning and at the middle point of the pumping cycle, which also challenges the adiabaticity of the pump. To prevent the condensate from expanding, we employ an additional harmonic optical potential

$$
V_{\mathrm{opt}}(r, \phi, z)=\frac{m}{2}\left(\omega_{r}^{2} r^{2}+\omega_{z}^{2} z^{2}\right),
$$

where $m$ is the atomic mass and $\omega_{k}$ the frequency of the potential along direction $k$. This kind of potential can be realized with laser fields [1].

We have studied in detail the temporal evolution of a $F=1$ condensate during vortex pumping by modeling it with the time-dependent Gross-Pitaevskii equation 23, $24]$

$$
\begin{aligned}
i \hbar \partial_{t} \Psi(\boldsymbol{r}) & =\left[-\frac{\hbar^{2}}{2 m} \nabla^{2}+V_{\mathrm{plug}}+V_{\mathrm{opt}}+\mu_{B} g_{F} \boldsymbol{B} \cdot \mathcal{F}\right. \\
& \left.+c_{0}|\boldsymbol{\Psi}|^{2}+c_{2} \mathcal{F} \cdot\left(\boldsymbol{\Psi}^{\dagger} \mathcal{F} \boldsymbol{\Psi}\right)\right] \boldsymbol{\Psi}(\boldsymbol{r})
\end{aligned}
$$

where $\boldsymbol{\Psi}(\boldsymbol{r})$ is a vector representation of the spinor order parameter $\langle\boldsymbol{r} \mid \Psi\rangle, \mu_{B}$ the Bohr magneton, $g_{F}=1 / 2$ the Landé $g$ factor, and $c_{0}=4 \pi \hbar^{2}\left(a_{0}+2 a_{2}\right) /(3 m)$ and $c_{2}=4 \pi \hbar^{2}\left(a_{2}-a_{0}\right) /(3 m)$ the coupling constants related to $s$-wave scattering lengths $a_{0}$ and $a_{2}$ of the atoms for different spin channels. We study condensates with repulsive atom-atom interactions, for which the atomspecific constant $c_{0}$ is positive. The constant $c_{2}$ is taken to be negative for the condensate to be ferromagnetic.
We consider a pancake-shaped condensate for which the $z$ dependence of the particle density can be taken to have the Gaussian form $e^{-z^{2} /\left(2 a_{z}^{2}\right)}$, where $a_{k}=$ $\sqrt{\hbar /\left(m \omega_{k}\right)}$ is the oscillator length in the direction $k$. This approximation is valid for $\omega_{z} \gg \omega_{r}$ and implies that the $z$ dependence of the GP equation can be factored out. We measure the length in the units of $a_{r}$, time in $1 / \omega_{r}$, energy in $\hbar \omega_{r}$, and magnetic field in $\hbar \omega_{r} / \mu_{B}$, and normalize the order parameter to unity. This results in a GP equation which depends only on the dimensionless parameters $\tilde{c}_{0}=2 \sqrt{2 \pi} N\left(a_{0}+2 a_{2}\right) /\left(3 a_{z}\right)$, $\tilde{c}_{2}=2 \sqrt{2 \pi} N\left(a_{2}-a_{0}\right) /\left(3 a_{z}\right), A /\left(\hbar \omega_{r}\right)$, and $\delta / a_{r}$, together with the time-dependent magnetic field.
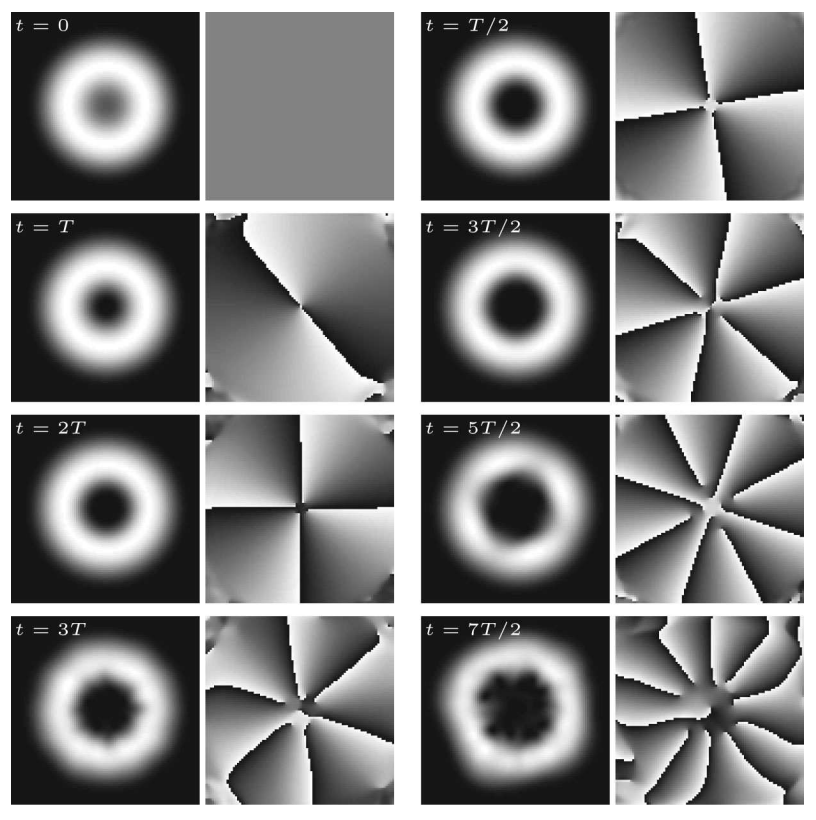

FIG. 2: Particle density and the complex phase of the $\mid F=$ $\left.1, m_{z}=-1\right\rangle$ (left columns) and $\left|F=1, m_{z}=1\right\rangle$ (right columns) components of the order parameter at half integer multiples of the pumping period $T=360 / \omega_{r}$. The field of view is $12 a_{r} \times 12 a_{r}$.

We choose the parameters used in our numerical computations according to ${ }^{87} \mathrm{Rb}[1]$, such that $c_{2} / c_{0}=-0.01$. Furthermore, we set $\tilde{c}_{0}=250$. We note that the atomatom interactions do not play a significant role in the operation of the vortex pump and qualitatively similar results are obtained, e.g., in the non-interacting case and for anti-ferromagnetic interactions. The parameters of the optical plug defined in Eq. (9) are chosen as $A=10 \hbar \omega_{r}$ and $\delta=2 a_{r}$. The maximum values of the magnetic fields are taken to be $B_{z}^{\max }=40 \hbar \omega_{r} / \mu_{B}$, $B_{q}^{\prime \max }=\hbar \omega_{r} /\left(\mu_{B} a_{r}\right)$, and $B_{h}^{\prime \max }=\hbar \omega_{r} /\left(\mu_{B} a_{r}\right)$.

Before beginning the pumping cycle, the ground state of the condensate is found by a relaxation method with the magnetic field parameters set to values corresponding to $t=0$ in Fig. 1, Figure 2 shows the particle density and the phase of the relevant components of the condensate during vortex pumping. The accumulation of 

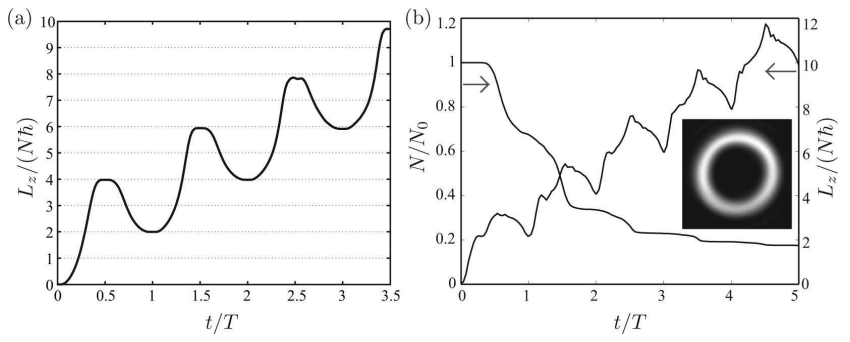

FIG. 3: Accumulation of axial angular momentum to the system during (a) adiabatic vortex pumping $\left(T=360 / \omega_{r}\right)$ and (b) pumping without any optical potentials $\left(T=10 / \omega_{r}\right)$, for which the fraction of atoms in the magnetic trap decreases in time as shown. The inset shows the particle density after five full cycles of non-adiabatic pumping with the field of view $12 a_{r} \times 12 a_{r}$.

two quanta of vorticity per cycle is clearly visible in the complex phase of the condensate. As more vortices are pumped to the BEC, the density depletion along the $z$ axis grows due to increasing core size of the vortex with increasing vorticity. The energetic and dynamic stability of the multi-quantum vortex is not guaranteed if the core area is much larger than the size of the optical plug, and hence initiation of splitting of the multi-quantum vortex into single-quantum vortices is observed for the ten-quantum vortex shown in Fig. 2. The observed instability can be overcome by increasing the strength and the width of the plug.

The number of vortices in each spin component is quantized, but the axial angular momentum is a continuous quantity as shown in Fig. 33(a). The figure illustrates the increment of vorticity by four during the field reversal in the presence of the hexapole field and the decrement of it by two when the field is brought back to its initial position with the quadrupole field turned on. The deviation of the axial angular momentum from $2 n \hbar N$ after $n$ pumping cycles is due to excitations away from the instantaneous eigenstate the pump is operated in.

Although the optical potentials given by Eqs. (9) and (10) are experimentally realizable using standard techniques, they can render the experiment rather com- plicated. However, the vortex pump can also be operated non-adiabatically without any optical potentials, since the spin components which do not follow the magnetic field are not trapped, and hence leave the condensate region. In fact, no optical fields were employed in the experiments of Ref. [19] with the cost of loosing roughly half of the atoms during the reversal of the bias field. Furthermore, if the maximum value of the bias field is much larger than that of the perpendicular field, an instantaneous switch between the quadrupole and hexapole field results in a negligible atom loss. Thus we suggest that the first experimental realization of the vortex pump could be in a set-up corresponding to the one used in Refs. 9, 19, 25 but with three additional coils to generate the hexapole field. Our numerical studies on vortex pumping with this technique show that in fact the total atom loss is considerably suppressed by the presence of the pumped multi-quantum vortex along the $z$ axis, causing the particle density to vanish there, see Fig. 3(b). Since the cycle can be traversed much faster than in the fully adiabatic case, there is no time for the multi-quantum vortex to split, and hence a clean tenquantum vortex is observed in the inset of Fig. 3(b) after five pumping cycles.

In conclusion, we have shown how to implement a vortex pump for spinor Bose-Einstein condensates using experimentally feasible techniques. Adiabatic and nonadiabatic operation of the pump was demonstrated computationally in the case of hyperfine spin $F=1$ by pumping vortices up to twelve circulation quanta to the condensate. In contrast to the experimentally created giant vortex [14], our scheme produces a pure multi-quantum vortex without accompanying single-quantum vortices. This vortex pump is an interesting example of adiabatic quantum dynamics for which the control parameters of the system are varied cyclically but the system does not return to its initial eigenspace. Our vortex pump paves the way for vortex-based applications of BECs since it can work as a vortex source, similar to a spin polarized current source in spintronics.

We acknowledge the Academy of Finland, Finnish Cultural Foundation, Wihuri foundation, and Väisälä foundation for financial support.
[1] C. J. Pethick and H. Smith, Bose-Einstein Condensation in Dilute Gases (Cambridge University Press, Cambridge, 2002).

[2] M. R. Matthews, B. P. Anderson, P. C. Haljan, D. S. Hall, C. E. Wieman, and E. A. Cornell, Phys. Rev. Lett. 83, 2498 (1999).

[3] J. E. Williams and M. J. Holland, Nature 401, 568 (1999).

[4] S. Inouye, S. Gupta, T. Rosenband, A. P. Chikkatur, A. Görlitz, T. L. Gustavson, A. E. Leanhardt, D. E. Pritchard, and W. Ketterle, Phys. Rev. Lett. 87, 080402 (2001).
[5] D. R. Scherer, C. N. Weiler, T. W. Neely, and B. P. Anderson, Phys. Rev. Lett. 98, 110402 (2007).

[6] K. W. Madison, F. Chevy, W. Wohlleben, and J. Dalibard, Phys. Rev. Lett. 84, 806 (2000).

[7] E. Hodby, G. Hechenblaikner, S. A. Hopkins, O. M. Maragò, and C. J. Foot, Phys. Rev. Lett. 88, 010405 (2001).

[8] M. Möttönen, T. Mizushima, T. Isoshima, M. M. Salomaa, and K. Machida, Phys. Rev. A 68, 023611 (2003).

[9] Y. Shin, M. Saba, M. Vengalattore, T. A. Pasquini, C. Sanner, A. E. Leanhardt, M. Prentiss, D. E. Pritchard, and W. Ketterle, Phys. Rev. Lett. 93, 160406 (2004). 
[10] J. A. M. Huhtamäki, M. Möttönen, T. Isoshima, V. Pietilä, and S. M. M. Virtanen, Phys. Rev. Lett. 97, 110406 (2006).

[11] J. R. Abo-Shaeer, C. Raman, J. M. Vogels, and W. Ketterle, Science 292, 476 (2001).

[12] C. Raman, J. R. Abo-Shaeer, J. M. Vogels, K. Xu, and W. Ketterle, Phys. Rev. Lett. 87, 210402 (2001).

[13] M. F. Andersen, C. Ryu, P. Cladé, V. Natarajan, A. Vaziri, K. Helmerson, and W. D. Phillips, Phys. Rev. Lett. 97, 170406 (2006).

[14] P. Engels, I. Coddington, P. C. Haljan, V. Schweikhard, and E. A. Cornell, Phys. Rev. Lett. 90, 170405 (2003).

[15] M. Nakahara, T. Isoshima, K. Machida, S.-I. Ogawa, and T. Ohmi, Physica B 284-288, 17 (2000).

[16] T. Isoshima, M. Nakahara, T. Ohmi, and K. Machida, Phys. Rev. A 61, 063610 (2000).

[17] S.-I. Ogawa, M. Möttönen, M. Nakahara, T. Ohmi, and H. Shimada, Phys. Rev. A 66, 013617 (2002).

[18] M. Möttönen, N. Matsumoto, M. Nakahara, and T. Ohmi, J. Phys.: Condens. Matter 14, 13481 (2002).
[19] A. E. Leanhardt, A. Görlitz, A. P. Chikkatur, D. Kielpinski, Y. Shin, D. E. Pritchard, and W. Ketterle, Phys. Rev. Lett. 89, 190403 (2002).

[20] N. R. Cooper, N. K. Wilkin, and J. M. F. Gunn, Phys. Rev. Lett. 87, 120405 (2001).

[21] M. V. Berry, Proc. R. Soc. London, Ser. A 392, 45 (1984).

[22] T. P. Simula, P. Engels, I. Coddington, V. Schweikhard, E. A. Cornell, and R. J. Ballagh, Phys. Rev. Lett. 94, 080404 (2005).

[23] T.-L. Ho, Phys. Rev. Lett. 81, 742 (1998).

[24] T. Ohmi and K. Machida, J. Phys. Soc. Jpn. 67, 1822 (1998).

[25] A. E. Leanhardt, Y. Shin, D. Kielpinski, D. E. Pritchard, and W. Ketterle, Phys. Rev. Lett. 90, 140403 (2003).

[26] Actually, anti-ferromagnetic atom-atom interactions can render the spin part of the condensate to deviate from this. Hence, we consider only the ferromagnetic case, although it is not crucial for the vortex pump to work. 